\title{
Hydrometrocolpos Presenting as Abdominal Distension in the Newborn
}

\author{
Arora $\mathrm{S}^{1}$, Narang $\mathrm{GS}^{2}$, Khurana $\mathrm{MS}^{3}$, Khera $\mathrm{A}^{4}$ \\ ${ }^{1}$ Dr. Sunita Arora, MBBS, MD, Associate Professor, \\ ${ }^{2}$ Dr. Gursharan Singh Narang, MBBS, MD, Professor, \\ ${ }^{3} \mathrm{Dr}$. Mandeep Singh Khurana, MBBS. MD, Associate \\ Professor, ${ }^{4} \mathrm{Dr}$. Anubha Khera, MBBS, Resident. All \\ from Sri Guru Ram Das Institute of Medical Sciences \\ and Research, Vallah, Amritsar, India.
}

\section{Introduction}

$\mathrm{O}$ bstruction of the vagina with accumulation of secretions and distention of the vagina was first reported in $1856^{1}$. When only the vagina is distended, it is termed hydrocolpos, but if there is associated uterine enlargement, the term hydrometrocolpos is applied. In the newborn, vaginal obstruction could result from high vaginal septum, varying degrees of vaginal atresia, cloacal malformation or an imperforate hymen ${ }^{2,3}$.

\section{The Case}

A two day old full term, appropriate for date, female child, born by LSCS presented to us with marked abdominal distension with mild respiratory distress since birth, non passage of urine but the child was passing stools normally. On physical examination, baby was active. There was generalized distension of abdomen and superficial veins were visible.(Figure-1).There was no hepatosplenomegaly and kidneys were not palpable. The baby passed urine adequately on catheterisation. Rest of the systemic examination was normal. There were no associated congenital anomalies and external genitalia apparently were normal.

Antenatal ultrasound revealed distended fetal abdomen with mild ascites bilateral hydronephrotic kidneys and a large cystic mass (approx $7.5 \times 7 \mathrm{~cm}$ ) in the lower abdomen lying adjacent to the urinary bladder.

On investigation, complete blood counts and serum electrolytes, were within normal limits but blood urea and serum creatinine were raised at presentation. X- ray abdomen in upright position revealed no air descended till the rectum. Abdominal ultrasound showed cystic

\section{Address for correspondence \\ Dr. Gursharan Singh Narang \\ E-mail: gsnarang321@gmail.com}

This work is licensed under a Creative Commons Attribution 3.0 License.

\begin{abstract}
Hydrometrocolpos is a rare entity in the newborn period with an incidence of 1 in 16000 female deliveries. We report a rare case of a two days old female child who presented to us with marked abdominal distension at birth due to hydrometrocolpos.
\end{abstract}

Key words: Hydrocolpos, Hydrometrocolpos, Cystic, Distension

mass extending from stomach upto the pelvis and bilateral kidneys revealed fullness of the pelvicalyceal system with dilatation of ureters along the entire course. Preoperatively we could not arrive at any diagnosis.

Emergency laparotomy was done and the uterus was found to be massively distended reaching upto epigastrium with adhesions of uterus with the omentum and surrounding gut loops and vaginal orifice was found to be absent. Stab incision was given over anterior wall of uterus, secretions were drained, vagina created by stitching labial mucosa to vaginal mucosa. Infant feeding tube No; 7(Figure-2) was passed through the uterine opening into newly created vagina which was left in situ. Postoperatively, blood urea and serum creatinine returned to normal.

The child had an uneventful recovery and serial vaginal dilatations were planned subsequently.

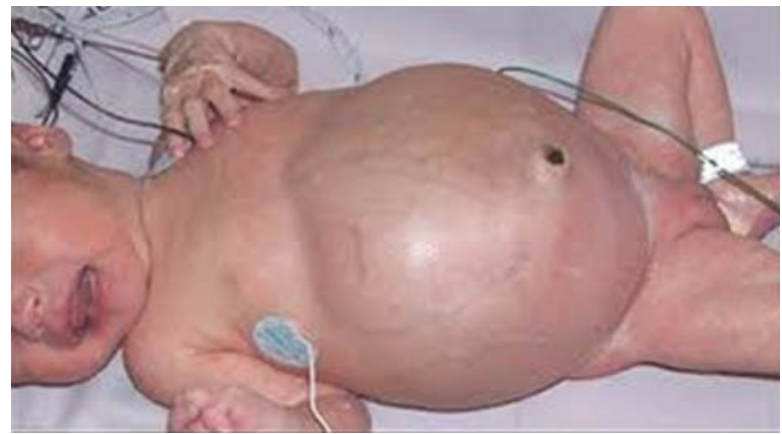

Fig 1: Preoperative; Marked abdominal distension with prominent superficial veins

How to cite this article?

Arora S, Narang GS, Khurana MS, Khera A. Hydrometrocolpos Presenting as Abdominal Distension in the Newborn. J Nepal Paediatr Soc 2013;33(2):136-137. 


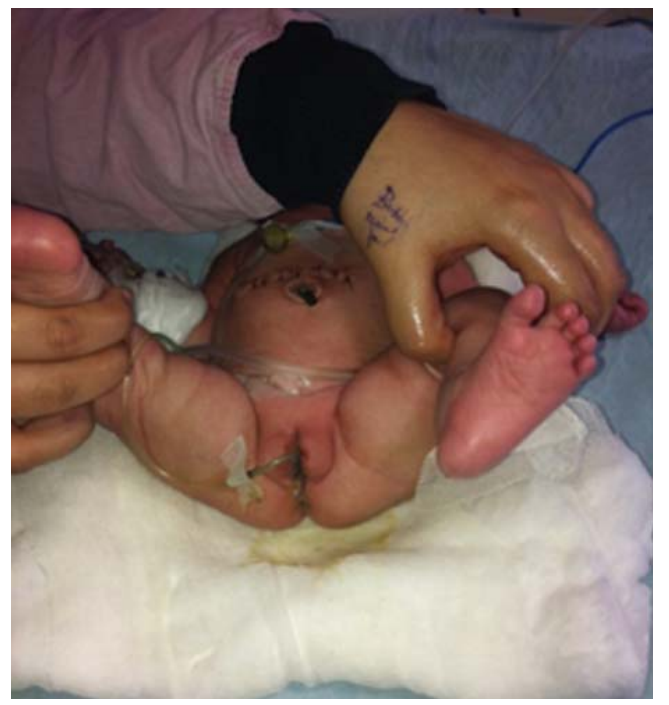

Fig 2: Postoperative; feeding tube negotiated through the uterine opening into newly created vagina.

\section{Discussion}

Hydrocolpos is an uncommon congenital disorder consisting of cystic dilatation of the vagina caused by obstruction of the distal genital tract, resulting in retained fluid. Hydrocolpos may manifest at birth as a palpable mass or it may present at puberty as progressive accumulation of menstrual blood, causing haematometrocolpos. Hydrocolpos and hydrometrocolpos are the third most common causes of all abdominal masses in the newborn, accounting for $15 \%$ of all abdominal masses in female newborns, with an estimated occurrence rate of 1 in 16,000 female deliveries ${ }^{3}$.

An overly distended vagina and uterus can compress the adjacent organs and cause abdominal pain, intestinal obstruction, ureteral obstruction, hydronephrosis, bladder perforation, respiratory distress, and lower extremity venous stasis. Accordingly, presentation of hydrometrocolpos depends on the degree of compression of the surrounding structures by the utero-vaginal swelling.

Maternal hormones profoundly affect the reproductive tract of the female infant both in utero and during the early neonatal period. In response to maternal oestrogens, vaginal and cervical epithelium secretes mucus, which pools in the obstructed vagina. Thus, hydrocolposis is usually detected in infancy when there is a high level of maternal estrogens

The McKusick-Kaufman syndrome is a rare autosomal recessive disorder mapped to 20p12 characterised by hydrometrocolpos, polydactyly, congenital heart defects ${ }^{4}$. The Bardet-Biedl syndrome is a genetic name for a heterogeneous group of autosomal recessive disorders with at least four loci in $16 q 13 q 22$, $11 q 13,3 p 11-p 13$, and $15 q 22.10$. It is characterised by retinal dystrophy or retinitis pigmentosa, postaxial polydactyly, obesity, nephropathy, and mental disturbances or mental retardation. It is also associated with hydrometrocolpos, usually as a consequence of vaginal atresia or transverse vaginal septum ${ }^{5,7}$. In a situation where hydrometrocolpos results from imperforate hymen, a tense protruding membrane is characteristically evident at the vulva obviating the need for laparotomy ${ }^{5}$.

Current management emphasizes prenatal diagnosis with ultrasound or magnetic resonance imaging ${ }^{3,7}$. The definitive treatment involves drainage of the accumulated fluid and establishing communication between the vaginal epithelium and the vulva ${ }^{4,5}$. For imperforate hymen and low vaginal atresia, perineal approach is preferable. Abdomino-perineal approach is usually reserved for high vaginal atresia ${ }^{7,8}$.

\section{Conclusion}

In our case the diagnosis could only be made intraoperatively. Possibility of hydrometrocolpos should be considered in any female newborn presenting with an abdominal mass of obscure origin. So, female genitalia should always be explored in every newborn presenting with abdominal distension.

\section{References}

1. Godefroy M. Imperforation de la membrane hymen produisant des accidents chez une enfant de deuxmoi. Operation GuerisonLancetteFr, 1856;29:567.

2. Ho NK.Hydocolpos.Aust NZ J. Obstet Gynaecol 1975;15:186-8.

3. Belghith M, Walha F, Saad H, Nouri A, Pouce H, Gueddiche N, et al. Neonatal hydrocolpos with distal vaginal atresia: Apropos of 2 cases. Chir Pediatr. 1990;31(6):352-3.

4. Alchihabi N, Pinter A. Surgical management of congenital high vaginal atresia. Orv Hetil. 1993;134:737-42.

5. Nazir Z, Rizvi RM, Qureshi RN, Khan ZS, Khan Z. Congenital vaginal obstructions: Varied presentation and outcome. Pediatr Surg Int.2006;22(9):749-53.

6. Gupta I, Barson AJ. Hydrocolpos with peritonitis in the newborn. J Clin Pathol. 1980;33(7):679-83.

7. David A, Bitoun P, Lacombe D, Lambert J, Nivelon $A$, Vigneron $\mathrm{J}$, et al . Hydrometrocolpos and polydactyly: A common neonatal presentation of Bardet-Biedl and McKusick-Kaufman syndromes. J Med Gent.1999;36:599-603.

8. Hahn-Pedersen J, Kvist $\mathrm{N}$, Nielsen $\mathrm{OH}$. Hydrometrocolpos: Current views on pathogenesis and management. J Urol.1984;132(3):537-40. 